\title{
Global stability of delayed virus infection model including multi-target cells and B-cell impairment
}

\author{
Safiya F. Alshehaiween ${ }^{\mathrm{a}}$, Ahmed M. Elaiw ${ }^{\mathrm{b}, \mathrm{c}, *}$ \\ ${ }^{a}$ Department of Mathematics, Faculty of Science, Taibah University, P.O. Box 344, Medina 42353, Saudi Arabia. \\ ${ }^{b}$ Department of Mathematics, Faculty of Science, King Abdulaziz University, P.O. Box 80203, Jeddah 21589, Saudi Arabia. \\ ${ }^{c}$ Department of Mathematics, Faculty of Science, Al-Azhar University, Assiut Branch, Assiut, Egypt.
}

\begin{abstract}
In this paper, we formulate a virus infection model with $n$ classes of target uninfected cells, $n$ classes of latent infected cells, $n$ classes of active infected cells, virus particles, and B cells. Three types of time delays and the impairment of B cells are involved. The Well-posedness of the model is demonstrated. Basic reproduction number of infection $\mathcal{R}_{0}>0$ is established, which determines the existence of equilibria as follows; when $\mathcal{R}_{0}$ is greater than unity, and then the model has two equilibria. Otherwise, the model has only a single equilibrium. The global stability of equilibria is proven using Lyapunov's direct method and applying LaSalle's invariance principle. To support our theoretical results, we have performed some numerical simulations in case of $n=2$ where the model can describe the HIV dynamics with two types of target cells, CD4 ${ }^{+}$T cells and macrophages.
\end{abstract}

Keywords: Virus dynamics, global stability, multi-target cells, impairment of B cells, time delay.

2020 MSC: 34D20, 34D23, 37N25, 92B05.

(C)2021 All rights reserved.

\section{Introduction}

In the last decades, an abundant various mathematical models of human viral infections have been proposed, modified and analyzed [5, 11, 13, 18, 19, 35, 39, 42, 46, 48, 49, 51]. These models can help researchers to (i) discover the interaction between virus particles and different types of human body cells; (ii) predict the future clinical status of patient; (iii) design an efficient drug dose; and (iv) improve a new treatment. The basic virus dynamics model was introduced in [38] which described the interaction between uninfected target cells $(U(t))$, infected cells $(A(t))$ and virus particles $(V(t))$ over time $t$. Since a part of the infected cells are passing through the latent stage (which is a stage that infected cells cannot produce any virions until they become active later), the basic model have been expanded to consider this stage of infection by taking into account the dynamics of latent infected cells $(L(t))$ in several works (see, e.g., $[21,28,40])$.

Once a virus enters a body, the innate immunity reacts against the attack and the adaptive immunity activates. The adaptive immunity is an important defense line against viruses because it has a specific

\footnotetext{
${ }^{*}$ Corresponding author

Email addresses: safiya.f.sh@gmail.com (Safiya F. Alshehaiween), a_m_elaiw@yahoo.com (Ahmed M. Elaiw)

doi: $10.22436 /$ jmcs.023.03.07
}

Received: 2020-08-26 Revised: 2020-09-10 Accepted: 2020-09-28 
response to any invader and has a memory cells to remember this invader for a quick response in the next time [43]. One of the adaptive immune system army is B cells which generate antibodies to capture the viruses to erase them from the body by other immune cells [39]. However, B cells could confront some factors that impair their functions such as malnutrition, aging, cytotoxic drugs, irradiation, trauma, tumors, some diseases, e.g., diabetes and immunosuppression by microbes, e.g., malaria, measles virus and HIV $[1,6,9,36]$. The impairment of immune response could lead to fatal complications to patient [43].

During the viral infection process, the time delays are inseparable to several phases of infection such as delays during cell division, delays of virus production and delays in activation of some medications from a prodrug to an active form and so on. Therefore, varied kinds of time lags have been inserted into virus dynamics models to depict the interactions between virus particles and body cells in more realistic way $[7,10,17,23-26,32,37,50]$.

Some viruses target more than one type of cells to take advantage of them to replicate themselves, e.g., HIV-1 invades CD4 ${ }^{+} \mathrm{T}$ cells and macrophages [41]. HTLV-1 preferentially infects CD4 ${ }^{+} \mathrm{T}$ cells, CD8 ${ }^{+}$ $\mathrm{T}$ cells, dendritic cells, and monocytes [30]. Chikungunya virus targets macrophages, fibroblasts and endothelial cells [4]. Thus, the basic virus infection model with multi-target cells has been introduced by Elaiw in [12] and then it has been extended to include the latent stage of infection in the following form:

$$
\begin{aligned}
\dot{U}_{i}(t) & =\rho_{i}-\gamma_{i} U_{i}(t)-\omega_{i} U_{i}(t) V(t), \\
\dot{L}_{i}(t) & =\omega_{i} U_{i}(t) V(t)-\left(\zeta_{i}+v_{i}\right) L_{i}(t), \\
\dot{A_{i}}(t) & =v_{i} L_{i}(t)-\beta_{i} A_{i}(t), \\
\dot{V}(t) & =\sum_{i=1}^{n} \varkappa_{i} A_{i}(t)-\xi V(t),
\end{aligned}
$$

where $i=1,2, \ldots, n$. These symbols $U_{i}(t), L_{i}(t)$ and $A_{i}(t)$ denote the populations of uninfected cells, latent infected cells and active infected cells of class $i$, respectively. $\rho_{i}$ and $\gamma_{i}$ represent the birth and death rate constants of the uninfected cells of class $i$, respectively. The infection rate is given by $\omega_{i} U_{i} V$, where $\omega_{i}$ is rate constant of the virus-target incidence. The latent infected cells of class $i$ are transmitted to active infected cells of class $i$ at rate $v_{i} L_{i}$ and die at rate $\zeta_{i} L_{i}$. The active infected cells of class $i$ and free virus particles die at rates $\beta_{i} A_{i}$ and $\xi V$, respectively. An active infected cells of class $i$ produce an average number $\varkappa_{i}$ of virus particles.

In [14], the authors have improved model (1.1) and involved the dynamics of B cell immunity as:

$$
\begin{aligned}
\dot{U}_{i}(t) & =\rho_{i}-\gamma_{i} u_{i}(t)-\omega_{i} u_{i}(t) V(t), \\
\dot{L}_{i}(t) & =\left(1-\chi_{i}\right) \omega_{i} u_{i}(t) V(t)-\left(\zeta_{i}+v_{i}\right) L_{i}(t), \\
\dot{A_{i}}(t) & =\chi_{i} \omega_{i} u_{i}(t) V(t)+v_{i} L_{i}(t)-\beta_{i} A_{i}(t), \\
\dot{V}(t) & =\sum_{i=1}^{n} \varkappa_{i} A_{i}(t)-\xi V(t)-\rho V(t) B(t), \\
\dot{B}(t) & =\alpha+\delta V(t) B(t)-\mu B(t) .
\end{aligned}
$$

The virus particles are attacked by antibodies at rate $\rho \mathrm{VB}$. The antibodies are produced at constant rate $\alpha$, proliferated at rate $\delta \mathrm{VB}$ and died at rate $\mu \mathrm{B}$. A fraction $\left(1-\chi_{i}\right)$ of infected target cells is assumed to be latent infected cells and the remaining $\chi_{i}$ becomes active infected cells, where $0<\chi_{i}<1$. Mathematicians have modified multi-target cells models and discussed them in several works (see, e.g., [44, 45, 47]).

We motivated and inspired by the previous works to introduce our virus dynamics model including: (i) multi-target cells, (ii) latent stage of infection, (iii) multiple time delays, (iv) B-cell impairment.

\section{The proposed model}

For the sake of understanding the effect of B cell functions impairment on interaction between $n$ classes of uninfected target cells $U_{i}(t), n$ classes of latent infected cells $L_{i}(t), n$ classes of active infected 
cells $A_{i}(t)$, virus particles $V(t)$ and $B$ cells $B(t)$, where $i=1,2, \ldots, n$, we propose a mathematical model consisting of $(3 n+2)$-differential equations as:

$$
\begin{aligned}
\dot{U}_{i} & =\rho_{i}-\gamma_{i} U_{i}-\omega_{i} U_{i} V, \\
\dot{L_{i}} & =\left(1-\chi_{i}\right) e^{-\tau_{i} \eta_{i}} \omega_{i} U_{i}\left(t-\tau_{i}\right) V\left(t-\tau_{i}\right)-\left(\zeta_{i}+v_{i}\right) L_{i}, \\
\dot{A_{i}} & =\chi_{i} e^{-\lambda_{i} \theta_{i}} \omega_{i} U_{i}\left(t-\lambda_{i}\right) V\left(t-\lambda_{i}\right)+v_{i} L_{i}-\beta_{i} A_{i}, \\
\dot{V} & =\sum_{i=1}^{n} \varkappa_{i} e^{-\pi_{i} \iota_{i}} A_{i}\left(t-\pi_{i}\right)-\xi V-\rho V B, \\
\dot{B} & =\varepsilon V-\mu B-\vartheta V B,
\end{aligned}
$$

where we use $U_{i}=U_{i}(t), L_{i}=L_{i}(t), A_{i}=A_{i}(t), V=V(t)$ and $B=B(t)$ for simplicity. The B cells decay at the rate $\vartheta \mathrm{VB}$ due to impairment factors. The time from virus enters a target cell of category $i$ to become latent infected is given by $\tau_{i}$, while $\lambda_{i}$ is the time between virus enters a target cell of category $i$ and the production of new virus particles from active infected cell of class $i$. The probability of latent and active infected cells surviving to the age of $\tau_{i}$ and $\lambda_{i}$ are represented by $e^{-\tau_{i} \eta_{i}}$ and $e^{-\lambda_{i} \theta_{i}}$, respectively, where $\eta_{i}$ and $\theta_{i}$ are constants. The parameter $\pi_{i}$ represents the time necessary for immature virus to become mature. The factor $e^{-\pi_{i} \iota_{i}}$ represents the probability of immature virus surviving to the age of $\pi_{i}$. All the parameters of the model are positive.

\section{Preliminaries}

The initial conditions for model (2.1) take the form:

$$
\left\{\begin{array}{l}
U_{i}(z)=\varphi_{i}(z), L_{i}(z)=\varphi_{i+n}(z), A_{i}(z)=\varphi_{i+2 n}(z), i=1,2, \ldots, n, \\
V(z)=\varphi_{3 n+1}(z), \quad B(z)=\varphi_{3 n+2}(z), \\
\varphi_{k}(z) \geqslant 0, \quad z \in[-T, 0], \quad k=1,2, \ldots, 3 n+2
\end{array}\right.
$$

where $T=\max \left\{\tau_{1}, \ldots, \tau_{n}, \lambda_{1}, \ldots, \lambda_{n}, \pi_{1}, \ldots, \pi_{n}\right\}, \varphi_{k}(z) \in \mathcal{C}\left([-T, 0], \mathbb{R}_{+}\right)$and $\mathcal{C}$ is the Banach space of continuous functions mapping the interval $[-T, 0]$ into $\mathbb{R}_{+}$with norm $\left\|\varphi_{k}\right\|=\sup _{-T \leqslant z \leqslant 0}\left|\varphi_{k}(z)\right|$ for $\varphi_{k} \in \mathcal{C}$. Now, by the standard theory of functional differential equations [31], we ensure that model (2.1) has a unique solution satisfying the initial conditions (3.1).

\subsection{Non-negativity and boundedness of solution}

In the following, we establish the non-negativity and boundedness of solutions of model (2.1).

Proposition 3.1. For $i=1,2, \ldots, n$, let $\left(U_{i}, L_{i}, A_{i}, V, B\right)$ be any solution of model (2.1) satisfying the initial conditions (3.1), then $\mathrm{U}_{i}, \mathrm{~L}_{i}, \mathrm{~A}_{i}, \mathrm{~V}$ and $\mathrm{B}$ are all non-negative and ultimately bounded for $\mathrm{t} \geqslant 0$.

Proof. From the first equation of model (2.1), we get $\dot{u}_{i} \mid u_{i=0}=\rho_{i}>0$, therefore $u_{i}>0$ for all $t \geqslant 0$. Further, for all $t \in[0, T]$, we have

$$
\begin{aligned}
& \mathrm{L}_{i}(\mathrm{t})=\varphi_{i+n}(0) e^{-\left(\zeta_{i}+v_{i}\right) t}+\left(1-\chi_{i}\right) e^{-\tau_{i} \eta_{i}} \omega_{i} \int_{0}^{t} e^{-\left(\zeta_{i}+v_{i}\right)(t-x)} u_{i}\left(x-\tau_{i}\right) V\left(x-\tau_{i}\right) d x \geqslant 0, \\
& A_{i}(t)=\varphi_{i+2 n}(0) e^{-\beta_{i} t}+\int_{0}^{t} e^{-\beta_{i}(t-x)}\left(\chi_{i} e^{-\lambda_{i} \theta_{i}} \omega_{i} U_{i}\left(x-\lambda_{i}\right) V\left(x-\lambda_{i}\right)+v_{i} L_{i}(x)\right) d x \geqslant 0, \\
& V(t)=\varphi_{3 n+1}(0) e^{-\int_{0}^{t}(\xi+\rho B(y)) d y}+\sum_{i=1}^{n} \varkappa_{i} e^{-\pi_{i} \iota_{i}} \int_{0}^{t} e^{-\int_{x}^{t}(\xi+\rho B(y)) d y} A_{i}\left(x-\pi_{i}\right) d x \geqslant 0, \\
& B(t)=\varphi_{3 n+2}(0) e^{-\int_{0}^{t}(\mu+\vartheta V(y)) d y}+\varepsilon \int_{0}^{t} e^{-\int_{x}^{t}(\mu+\vartheta V(y)) d y} V(x) d x \geqslant 0 .
\end{aligned}
$$

By a recursive argument, we have $L_{i} \geqslant 0, A_{i} \geqslant 0, V \geqslant 0$ and $B \geqslant 0$ for all $t \geqslant 0$. Thus, we ensure 
the solutions of model (2.1) are non-negative. In order to show the boundedness of solutions, we have $\dot{U}_{i} \leqslant \rho_{i}-\gamma_{i} U_{i}$ from the first equation of model (2.1) which implies that $\lim _{t \rightarrow \infty} \sup _{i}(t) \leqslant \rho_{i} / \gamma_{i}$ and then $U_{i}$ is ultimately bounded. Now, let us consider

$$
F_{i}(t)=\left(1-\chi_{i}\right) e^{-\tau_{i} \eta_{i}} U_{i}\left(t-\tau_{i}\right)+\chi_{i} e^{-\lambda_{i} \theta_{i}} U_{i}\left(t-\lambda_{i}\right)+L_{i}+A_{i}, i=1,2, \ldots, n,
$$

then a time derivative of $F_{i}$ is given by:

$$
\begin{aligned}
\dot{F}_{i}= & \left(1-\chi_{i}\right) e^{-\tau_{i} \eta_{i}} \rho_{i}-\left(1-\chi_{i}\right) e^{-\tau_{i} \eta_{i}} \gamma_{i} u_{i}\left(t-\tau_{i}\right)-\left(1-\chi_{i}\right) e^{-\tau_{i} \eta_{i}} \omega_{i} u_{i}\left(t-\tau_{i}\right) V\left(t-\tau_{i}\right) \\
& +\chi_{i} e^{-\lambda_{i} \theta_{i}} \rho_{i}-\chi_{i} e^{-\lambda_{i} \theta_{i}} \gamma_{i} u_{i}\left(t-\lambda_{i}\right)-\chi_{i} e^{-\lambda_{i} \theta_{i}} \omega_{i} u_{i}\left(t-\lambda_{i}\right) V\left(t-\lambda_{i}\right) \\
& +\left(1-\chi_{i}\right) e^{-\tau_{i} \eta_{i}} \omega_{i} u_{i}\left(t-\tau_{i}\right) V\left(t-\tau_{i}\right)-\left(\tau_{i}+v_{i}\right) L_{i}+\chi_{i} e^{-\lambda_{i} \theta_{i}} \omega_{i} u_{i}\left(t-\lambda_{i}\right) V\left(t-\lambda_{i}\right)+v_{i} L_{i}-\beta_{i} A_{i} \\
= & \left(1-\chi_{i}\right) e^{-\tau_{i} \eta_{i}} \rho_{i}-\left(1-\chi_{i}\right) e^{-\tau_{i} \eta_{i}} \gamma_{i} u_{i}\left(t-\tau_{i}\right)+\chi_{i} e^{-\lambda_{i} \theta_{i}} \rho_{i}-\chi_{i} e^{-\lambda_{i} \theta_{i}} \gamma_{i} u_{i}\left(t-\lambda_{i}\right)-\zeta_{i} L_{i}-\beta_{i} A_{i} \\
\leqslant & \rho_{i}-\sigma_{i}\left[\left(1-\chi_{i}\right) e^{-\tau_{i} \eta_{i}} u_{i}\left(t-\tau_{i}\right)+\chi_{i} e^{-\lambda_{i} \theta_{i}} u_{i}\left(t-\lambda_{i}\right)+L_{i}+A_{i}\right] \\
= & \rho_{i}-\sigma_{i} F_{i}(t),
\end{aligned}
$$

where $\sigma_{i}=\min \left\{\gamma_{i}, \zeta_{i}, \beta_{i}\right\}$. It follows that

$$
\lim _{t \rightarrow \infty} \sup F_{i}(t) \leqslant c_{i}, \quad \lim _{t \rightarrow \infty} \sup L_{i}(t) \leqslant c_{i}, \quad \lim _{t \rightarrow \infty} \sup A_{i}(t) \leqslant c_{i},
$$

where $c_{i}=\rho_{i} / \sigma_{i}$. Now, let $H(t)=V+\frac{\xi}{2 \varepsilon} B$, then we obtain

$$
\begin{aligned}
\dot{H} & =\sum_{i=1}^{n} \varkappa_{i} e^{-\pi_{i} \iota_{i}} A_{i}\left(t-\pi_{i}\right)-\xi V-\rho V B+\frac{\xi}{2} V-\frac{\xi \mu}{2 \varepsilon} B-\frac{\xi \vartheta}{2 \varepsilon} V B \\
& =\sum_{i=1}^{n} \varkappa_{i} e^{-\pi_{i} \iota_{i}} A_{i}\left(t-\pi_{i}\right)-\frac{\xi}{2} V-\frac{\xi \mu}{2 \varepsilon} B-\left(\rho+\frac{\xi \vartheta}{2 \varepsilon}\right) V B \\
& \leqslant \sum_{i=1}^{n} \varkappa_{i} e^{-\pi_{i} \iota_{i}} c_{i}-\frac{\xi}{2} V-\frac{\xi \mu}{2 \varepsilon} B \\
& \leqslant \sum_{i=1}^{n} \varkappa_{i} e^{-\pi_{i} \iota_{i}} c_{i}-\ell\left(V+\frac{\xi}{2 \varepsilon} B\right) \\
& =\sum_{i=1}^{n} \varkappa_{i} e^{-\pi_{i} \iota_{i}} c_{i}-\ell H,
\end{aligned}
$$

where $\ell=\min \left\{\frac{\xi}{2}, \mu\right\}$. In a similar way, we have $\lim _{t \rightarrow \infty} \sup V(t) \leqslant \bar{c}$ and $\lim _{t \rightarrow \infty} \sup B(t) \leqslant \widetilde{c}$, where $\bar{c}$ $=\frac{\sum_{i=1}^{n} \varkappa_{i} e^{-\pi_{i} \iota_{i} c_{i}}}{\ell}$ and $\widetilde{c}=\frac{2 \varepsilon \bar{c}}{\xi}$. Thus, the solutions of model (2.1) are ultimately bounded.

The previous proof reveals that $\Omega$ limit sets of model (2.1) are contained in the following bounded feasible region $\Gamma$

$$
\Gamma=\left\{\left(\mathrm{U}_{i}, \mathrm{~L}_{\mathrm{i}}, \mathrm{A}_{\mathrm{i}}, \mathrm{V}, \mathrm{B}\right) \in \mathbb{R}_{+}^{3 n+2}:\left\|\mathrm{U}_{i}\right\| \leqslant \frac{\rho_{i}}{\gamma_{i}},\left\|\mathrm{~L}_{i}\right\| \leqslant \mathrm{c}_{i},\left\|A_{i}\right\| \leqslant \mathrm{c}_{i},\|\mathrm{~V}\| \leqslant \overline{\mathrm{c}},\|\mathrm{B}\| \leqslant \widetilde{\mathrm{c}}\right\},
$$

which implies that the solutions of model (2.1) point towards the region $\Omega$. For this reason, $\Omega$ is positively invariant for model (2.1).

\subsection{Equilibria}

The basic reproduction number of infection for model (2.1) is

$$
\mathrm{R}_{0}=\sum_{i=1}^{n} \mathrm{R}_{0 i}=\sum_{i=1}^{n} \frac{\rho_{i}\left(\psi_{i}+\phi_{i}\right)}{\xi \gamma_{i}},
$$

where $\psi_{i}=\frac{\varkappa_{i} \omega_{i} \nu_{i}\left(1-\chi_{i}\right) e^{-\pi_{i} \iota_{i}-\tau_{i} \eta_{i}}}{\beta_{i}\left(\zeta_{i}+v_{i}\right)}$ and $\phi_{i}=\frac{\varkappa_{i} \omega_{i} \chi_{i} e^{-\pi_{i} \iota_{i}-\lambda_{i} \theta_{i}}}{\beta_{i}}$. 
Lemma 3.2. Consider model (2.1), then

(i) the model has a unique equilibrium $\mathrm{E}^{0}$ if $\mathrm{R}_{0} \leqslant 1$;

(ii) the model has two equilibria $\mathrm{E}^{0}$ and $\mathrm{E}^{*}$ if $\mathrm{R}_{0}>1$.

Proof. At any equilibrium, we have

$$
\begin{aligned}
& \rho_{i}-\gamma_{i} U_{i}-\omega_{i} U_{i} V=0, \\
&\left(1-\chi_{i}\right) e^{-\tau_{i} \eta_{i}} \omega_{i} U_{i} V-\left(\zeta_{i}+v_{i}\right) L_{i}=0, \\
& \chi_{i} e^{-\lambda_{i} \theta_{i}} \omega_{i} U_{i} V+v_{i} L_{i}-\beta_{i} A_{i}=0,=0, \\
& \sum_{i=1}^{n} \varkappa_{i} e^{-\pi_{i} \iota_{i}} A_{i}-\xi V-\rho V B=0 \\
& \varepsilon V-\mu B-\vartheta V B=0 .
\end{aligned}
$$

We obtain $U_{i}, L_{i}, A_{i}$ and $B$ as functions of $V$ as follow:

$$
\begin{aligned}
\mathrm{u}_{i} & =\frac{\rho_{i}}{\gamma_{i}+\omega_{i} V^{\prime}} \\
\mathrm{L}_{i} & =\frac{e^{-\tau_{i} \eta_{i}} \omega_{i} \rho_{i}\left(1-\chi_{i}\right) \mathrm{V}}{\left(\zeta_{i}+v_{i}\right)\left(\gamma_{i}+\omega_{i} V\right)}, \\
A_{i} & =\frac{\omega_{i} \rho_{i}\left(\nu_{i}\left(1-\chi_{i}\right) e^{-\tau_{i} \eta_{i}}+\chi_{i} e^{-\lambda_{i} \theta_{i}}\left(\zeta_{i}+v_{i}\right)\right) V}{\beta_{i}\left(\zeta_{i}+\nu_{i}\right)\left(\gamma_{i}+\omega_{i} V\right)}, \\
B & =\frac{\varepsilon V}{\mu+\vartheta V} .
\end{aligned}
$$

Substituting Eqs. (3.4) and (3.5) into Eq. (3.2), then we get

$$
\sum_{i=1}^{n} \frac{\varkappa_{i} \omega_{i} \rho_{i} e^{-\pi_{i} \iota_{i}}\left(\nu_{i}\left(1-\chi_{i}\right) e^{-\tau_{i} \eta_{i}}+\chi_{i} e^{-\lambda_{i} \theta_{i}}\left(\zeta_{i}+\nu_{i}\right)\right) V}{\beta_{i}\left(\zeta_{i}+v_{i}\right)\left(\gamma_{i}+\omega_{i} V\right)}-\xi V-\frac{\rho \varepsilon V^{2}}{\mu+\vartheta V}=0 .
$$

Clearly, $V=0$ is a solution of Eq. (3.6), therefore we have the infection-free equilibrium $E^{0}=\left(U_{i}^{0}, 0,0,0,0\right)$, where $U_{i}^{0}=\frac{\rho_{i}}{\gamma_{i}}$. The other possibility of Eq. (3.6) is

$$
\sum_{i=1}^{n} \frac{\varkappa_{i} \omega_{i} \rho_{i} e^{-\pi_{i} \iota_{i}}\left(\nu_{i}\left(1-\chi_{i}\right) e^{-\tau_{i} \eta_{i}}+\chi_{i} e^{-\lambda_{i} \theta_{i}}\left(\zeta_{i}+v_{i}\right)\right)}{\beta_{i}\left(\zeta_{i}+v_{i}\right)\left(\gamma_{i}+\omega_{i} V\right)}-\xi-\frac{\rho \varepsilon V}{\mu+\vartheta V}=0
$$

Let us define a function $Y(V)$ as:

$$
Y(V)=\sum_{i=1}^{n} \frac{\varkappa_{i} \omega_{i} \rho_{i} e^{-\pi_{i} \iota_{i}}\left(\nu_{i}\left(1-\chi_{i}\right) e^{-\tau_{i} \eta_{i}}+\chi_{i} e^{-\lambda_{i} \theta_{i}}\left(\zeta_{i}+\nu_{i}\right)\right)}{\beta_{i}\left(\zeta_{i}+\nu_{i}\right)\left(\gamma_{i}+\omega_{i} V\right)}-\xi-\frac{\rho \varepsilon V}{\mu+\vartheta V} .
$$

It is seen that $Y(V)$ is monotonic decreasing since

$$
Y^{\prime}(V)=-\left(\sum_{i=1}^{n} \frac{\varkappa_{i} \omega_{i}^{2} \rho_{i} e^{-\pi_{i} \iota_{i}}\left(\nu_{i}\left(1-\chi_{i}\right) e^{-\tau_{i} \eta_{i}}+\chi_{i} e^{-\lambda_{i} \theta_{i}}\left(\zeta_{i}+\nu_{i}\right)\right)}{\beta_{i}\left(\zeta_{i}+\nu_{i}\right)\left(\gamma_{i}+\omega_{i} V\right)^{2}}+\frac{\varepsilon \rho \mu}{(\mu+\vartheta V)^{2}}\right) .
$$

On the other hand, $\lim _{V \rightarrow \infty} Y(V)=-\frac{\xi \vartheta+\rho \varepsilon}{\vartheta}$. Further,

$$
Y(0)=\sum_{i=1}^{n} \frac{\varkappa_{i} \omega_{i} \rho_{i} e^{-\pi_{i} \iota_{i}}\left(\nu_{i}\left(1-\chi_{i}\right) e^{-\tau_{i} \eta_{i}}+\chi_{i} e^{-\lambda_{i} \theta_{i}}\left(\zeta_{i}+\nu_{i}\right)\right)}{\beta_{i} \gamma_{i}\left(\zeta_{i}+v_{i}\right)}-\xi=\xi\left(R_{0}-1\right) .
$$


It implies that $Y(V)$ has a unique positive solution $V^{*}$ if $R_{0}>1$. Thus, we have the endemic-infection equilibrium $E^{*}=\left(U_{i}^{*}, L_{i}^{*}, A_{i}^{*}, V^{*}, B^{*}\right)$, where

$$
\begin{array}{rlrl}
\mathrm{U}_{i}^{*} & =\frac{\rho_{\mathrm{i}}}{\gamma_{\mathrm{i}}+\omega_{i} \mathrm{~V}^{*}}, & \mathrm{~L}_{\mathrm{i}}^{*} & =\frac{e^{-\tau_{i} \eta_{i}} \omega_{i} \rho_{i}\left(1-\chi_{i}\right) \mathrm{V}^{*}}{\left(\zeta_{i}+\nu_{i}\right)\left(\gamma_{i}+\omega_{i} \mathrm{~V}^{*}\right)}, \\
A_{i}^{*}=\frac{\omega_{i} \rho_{i}\left(\nu_{i}\left(1-\chi_{i}\right) e^{-\tau_{i} \eta_{i}}+\chi_{i} e^{-\lambda_{i} \theta_{i}}\left(\zeta_{i}+\nu_{i}\right)\right) V^{*}}{\beta_{i}\left(\zeta_{i}+\nu_{i}\right)\left(\gamma_{i}+\omega_{i} V^{*}\right)} & B^{*}=\frac{\varepsilon V^{*}}{\mu+\vartheta V^{*}} .
\end{array}
$$

Therefore, we have proved the existence of endemic-infection equilibrium $E^{*}$ under the condition $R_{0}>$ 1.

\section{Global stability of equilibria}

In the following, we demonstrate the global stability of equilibria according to the value of $R_{0}$. To state the global stability results, we need to define a function $G(q)=q-1-\ln (q)$, where $q>0$.

Theorem 4.1. If $\mathrm{R}_{0}<1$, then the equilibrium $\mathrm{E}^{0}$ is globally asymptotically stable (G.A.S).

Proof. Construct a candidate Lyapunov functional as:

$$
\begin{aligned}
M_{0}= & \sum_{i=1}^{n} \frac{\psi_{i}+\phi_{i}}{\omega_{i}} U_{i}^{0} G\left(\frac{U_{i}}{U_{i}^{0}}\right)+\sum_{i=1}^{n} \frac{v_{i} \varkappa_{i} e^{-\pi_{i} \iota_{i}}}{\beta_{i}\left(\zeta_{i}+v_{i}\right)} L_{i}+\sum_{i=1}^{n} \frac{\varkappa_{i} e^{-\pi_{i} \iota_{i}}}{\beta_{i}} A_{i}+V \\
& +\frac{\xi\left(1-R_{0}\right)}{\varepsilon} B+\sum_{i=1}^{n} \psi_{i} \int_{t-\tau_{i}}^{t} U_{i}(x) V(x) d x+\sum_{i=1}^{n} \phi_{i} \int_{t-\lambda_{i}}^{t} U_{i}(x) V(x) d x+\sum_{i=1}^{n} \varkappa_{i} e^{-\pi_{i} \iota_{i}} \int_{t-\pi_{i}}^{t} A_{i}(x) d x .
\end{aligned}
$$

It's obvious that $M_{0}>0$ for all $\left(U_{i}, L_{i}, A_{i}, V, B\right)>0$ and $M_{0}=0$ at $E^{0}$. Now, the time derivative of $M_{0}$ along the solutions of model (2.1) is given by:

$$
\begin{aligned}
\frac{d M_{0}}{d t}= & \sum_{i=1}^{n} \frac{\psi_{i}+\phi_{i}}{\omega_{i}}\left(1-\frac{u_{i}^{0}}{u_{i}}\right)\left(\rho_{i}-\gamma_{i} u_{i}-\omega_{i} U_{i} V\right) \\
& +\sum_{i=1}^{n} \frac{v_{i} \varkappa_{i} e^{-\pi_{i} \iota_{i}}}{\beta_{i}\left(\zeta_{i}+v_{i}\right)}\left(\left(1-\chi_{i}\right) e^{-\tau_{i} \eta_{i}} \omega_{i} U_{i}\left(t-\tau_{i}\right) V\left(t-\tau_{i}\right)-\left(\zeta_{i}+v_{i}\right) L_{i}\right) \\
& +\sum_{i=1}^{n} \frac{\varkappa_{i} e^{-\pi_{i} \iota_{i}}}{\beta_{i}}\left(\chi_{i} e^{-\lambda_{i} \theta_{i}} \omega_{i} u_{i}\left(t-\lambda_{i}\right) V\left(t-\lambda_{i}\right)+v_{i} L_{i}-\beta_{i} A_{i}\right) \\
& +\sum_{i=1}^{n} \varkappa_{i} e^{-\pi_{i} \iota_{i}} A_{i}\left(t-\pi_{i}\right)-\xi V-\rho V B+\frac{\xi\left(1-R_{0}\right)}{\varepsilon}(\varepsilon V-\mu B-\vartheta V B) \\
& +\sum_{i=1}^{n} \psi_{i}\left(U_{i} V-U_{i}\left(t-\tau_{i}\right) V\left(t-\tau_{i}\right)\right)+\sum_{i=1}^{n} \phi_{i}\left(U_{i} V-U_{i}\left(t-\lambda_{i}\right) V\left(t-\lambda_{i}\right)\right) \\
& +\sum_{i=1}^{n} \varkappa_{i} e^{-\pi_{i} \iota_{i}}\left(A_{i}-A_{i}\left(t-\pi_{i}\right)\right) \\
= & -\sum_{i=1}^{n} \frac{\gamma_{i}\left(\psi_{i}+\phi_{i}\right)\left(U_{i}-U_{i}^{0}\right)^{2}}{\omega_{i} U_{i}}-\frac{\xi \mu\left(1-R_{0}\right)}{\varepsilon} B-\left(\rho+\frac{\vartheta \xi\left(1-R_{0}\right)}{\varepsilon}\right) V B .
\end{aligned}
$$

Thus $\frac{d M_{0}}{d t} \leqslant 0$ as long as $R_{0}<1$. Moreover, $\frac{d M_{0}}{d t}=0$ when $u_{i}(t)=u_{i}^{0}$ and $B(t)=0$. Let $\Delta=$ $\left\{\left(U_{i}, L_{i}, A_{i}, V, B\right): \frac{d M_{0}}{d t}=0\right\}$ and $\bar{\Delta}$ be the largest invariant subset of $\Delta$. Thus, the trajectories of model 
(2.1) tend to $\bar{\Delta}$ where every components of $\bar{\Delta}$ satisfy $\mathrm{U}_{i}(\mathrm{t})=\mathrm{U}_{i}^{0}$ and $\mathrm{B}(\mathrm{t})=0$. Then, the last equation of model (2.1) yields

$$
\dot{\mathrm{B}}(\mathrm{t})=0=\varepsilon \mathrm{V}(\mathrm{t}) \quad \Longrightarrow \quad \mathrm{V}(\mathrm{t})=0 .
$$

From the $4^{\text {th }}$ equation of model (2.1), we obtain

$$
\dot{V}(t)=0=\sum_{i=1}^{n} \varkappa_{i} e^{-\pi_{i} \iota_{i}} A_{i}\left(t-\pi_{i}\right) \quad \Longrightarrow \quad A_{i}(t)=0 .
$$

Similarly, from the $3^{\text {th }}$ equation of model (2.1), we get $L_{\mathfrak{i}}(t)=0$. Immediately, we can see that $\bar{\Delta}$ contains a single point $E^{0}$. Thus, according to LaSalle's invariance principle (L.I.P) [34], $E^{0}$ is G.A.S if $R_{0}<1$.

Theorem 4.2. If $\mathrm{R}_{0}>1$, then the equilibrium $\mathrm{E}^{*}$ is G.A.S.

Proof. Construct a candidate Lyapunov functional as:

$$
\begin{aligned}
M_{1}= & \sum_{i=1}^{n} \frac{\psi_{i}+\phi_{i}}{\omega_{i}} U_{i}^{*} G\left(\frac{U_{i}}{U_{i}^{*}}\right)+\sum_{i=1}^{n} \frac{v_{i} \varkappa_{i} e^{-\pi_{i} \iota_{i}}}{\beta_{i}\left(\zeta_{i}+v_{i}\right)} L_{i}^{*} G\left(\frac{L_{i}}{L_{i}^{*}}\right) \\
& +\sum_{i=1}^{n} \frac{\varkappa_{i} e^{-\pi_{i} \iota_{i}}}{\beta_{i}} A_{i}^{*} G\left(\frac{A_{i}}{A_{i}^{*}}\right)+V^{*} G\left(\frac{V}{V^{*}}\right)+\frac{\rho}{2\left(\varepsilon-\vartheta B^{*}\right)}\left(B-B^{*}\right)^{2} \\
& +\sum_{i=1}^{n} \psi_{i} U_{i}^{*} V^{*} \int_{t-\tau_{i}}^{t} G\left(\frac{U_{i}(x) V(x)}{U_{i}^{*} V^{*}}\right) d x+\sum_{i=1}^{n} \phi_{i} U_{i}^{*} V^{*} \int_{t-\lambda_{i}}^{t} G\left(\frac{U_{i}(x) V(x)}{U_{i}^{*} V^{*}}\right) d x \\
& +\sum_{i=1}^{n} \varkappa_{i} e^{-\pi_{i} \iota_{i}} A_{i}^{*} \int_{t-\pi_{i}}^{t} G\left(\frac{A_{i}(x)}{A_{i}^{*}}\right) d x .
\end{aligned}
$$

It is obvious from Eq. (3.3) that $\varepsilon-\vartheta B^{*}=\frac{\mu B^{*}}{V^{*}}>0$. Thus $M_{1}>0$ for all $\left(U_{i}, L_{i}, A_{i}, V, B\right)>0$ and $M_{1}=0$ at $E^{*}$. Now, the time derivative of $M_{1}$ is:

$$
\begin{aligned}
& \frac{d M_{1}}{d t}=\frac{\psi_{i}+\phi_{i}}{\omega_{i}}\left(1-\frac{u_{i}^{*}}{u_{i}}\right)\left(\rho_{i}-\gamma_{i} u_{i}-\omega_{i} U_{i} V\right) \\
& +\sum_{i=1}^{n} \frac{v_{i} \varkappa_{i} e^{-\pi_{i} \iota_{i}}}{\beta_{i}\left(\zeta_{i}+v_{i}\right)}\left(1-\frac{L_{i}^{*}}{L_{i}}\right)\left(\left(1-\chi_{i}\right) e^{-\tau_{i} \eta_{i}} \omega_{i} u_{i}\left(t-\tau_{i}\right) V\left(t-\tau_{i}\right)-\left(\zeta_{i}+v_{i}\right) L_{i}\right) \\
& +\sum_{i=1}^{n} \frac{\varkappa_{i} e^{-\pi_{i} \iota_{i}}}{\beta_{i}}\left(1-\frac{A_{i}^{*}}{A_{i}}\right)\left(\chi_{i} e^{-\lambda_{i} \theta_{i}} \omega_{i} U_{i}\left(t-\lambda_{i}\right) V\left(t-\lambda_{i}\right)+v_{i} L_{i}-\beta_{i} A_{i}\right) \\
& +\left(1-\frac{V^{*}}{V}\right)\left(\sum_{i=1}^{n} \varkappa_{i} e^{-\pi_{i} \iota_{i}} A_{i}\left(t-\pi_{i}\right)-\xi V-\rho V B\right)+\frac{\rho}{\varepsilon-\vartheta B^{*}}\left(B-B^{*}\right)(\varepsilon V-\mu B-\vartheta V B) \\
& +\sum_{i=1}^{n} \psi_{i} U_{i}^{*} V^{*}\left[\frac{U_{i} V}{U_{i}^{*} V^{*}}-\frac{U_{i}\left(t-\tau_{i}\right) V\left(t-\tau_{i}\right)}{U_{i}^{*} V^{*}}+\ln \left(\frac{U_{i}\left(t-\tau_{i}\right) V\left(t-\tau_{i}\right)}{U_{i} V}\right)\right] \\
& +\sum_{i=1}^{n} \phi_{i} u_{i}^{*} V^{*}\left[\frac{U_{i} V}{u_{i}^{*} V^{*}}-\frac{u_{i}\left(t-\lambda_{i}\right) V\left(t-\lambda_{i}\right)}{u_{i}^{*} V^{*}}+\ln \left(\frac{u_{i}\left(t-\lambda_{i}\right) V\left(t-\lambda_{i}\right)}{U_{i} V}\right)\right] \\
& +\sum_{i=1}^{n} \varkappa_{i} e^{-\pi_{i} \iota_{i}} A_{i}^{*}\left[\frac{A_{i}}{A_{i}^{*}}-\frac{A_{i}\left(t-\pi_{i}\right)}{A_{i}^{*}}+\ln \left(\frac{A_{i}\left(t-\pi_{i}\right)}{A_{i}}\right)\right] \\
& =\sum_{i=1}^{n} \frac{\psi_{i}+\phi_{i}}{\omega_{i}}\left(1-\frac{u_{i}^{*}}{u_{i}}\right)\left(\rho_{i}-\gamma_{i} u_{i}\right)+\sum_{i=1}^{n} \frac{\psi_{i}+\phi_{i}}{\omega_{i}} \omega_{i} u_{i}^{*} V
\end{aligned}
$$




$$
\begin{aligned}
& -\sum_{i=1}^{n} \psi_{i} u_{i}\left(t-\tau_{i}\right) V\left(t-\tau_{i}\right) \frac{L_{i}^{*}}{L_{i}}+\sum_{i=1}^{n} \frac{v_{i} \varkappa_{i} e^{-\pi_{i} \iota_{i}}}{\beta_{i}} L_{i}^{*} \\
& -\sum_{i=1}^{n} \phi_{i} U_{i}\left(t-\lambda_{i}\right) V\left(t-\lambda_{i}\right) \frac{A_{i}^{*}}{A_{i}}-\sum_{i=1}^{n} \frac{\varkappa_{i} e^{-\pi_{i} \iota_{i}}}{\beta_{i}} v_{i} L_{i} \frac{A_{i}^{*}}{A_{i}} \\
& +\sum_{i=1}^{n} \varkappa_{i} e^{-\pi_{i} \iota_{i}} A_{i}^{*}-\frac{V^{*}}{V} \sum_{i=1}^{n} \varkappa_{i} e^{-\pi_{i} \iota_{i}} A_{i}\left(t-\pi_{i}\right)-\xi\left(V-V^{*}\right)-\rho\left(V-V^{*}\right) B \\
& +\frac{\rho}{\varepsilon-\vartheta B^{*}}\left(B-B^{*}\right)(\varepsilon V-\mu B-\vartheta V B)+\sum_{i=1}^{n} \psi_{i} U_{i}^{*} V^{*} \ln \left(\frac{U_{i}\left(t-\tau_{i}\right) V\left(t-\tau_{i}\right)}{U_{i} V}\right) \\
& +\sum_{i=1}^{n} \phi_{i} U_{i}^{*} V^{*} \ln \left(\frac{U_{i}\left(t-\lambda_{i}\right) V\left(t-\lambda_{i}\right)}{U_{i} V}\right)+\sum_{i=1}^{n} \varkappa_{i} e^{-\pi_{i} \iota_{i}} A_{i}^{*} \ln \left(\frac{A_{i}\left(t-\pi_{i}\right)}{A_{i}}\right) .
\end{aligned}
$$

Utilizing the endemic-infection equilibrium conditions

$$
\begin{array}{rlrl}
\rho_{i} & =\gamma_{i} U_{i}^{*}+\omega_{i} U_{i}^{*} V^{*}, & L_{i}^{*}=\frac{\left(1-\chi_{i}\right) e^{-\tau_{i} \eta_{i}} \omega_{i} U_{i}^{*} V^{*}}{\left(\zeta_{i}+v_{i}\right)}, \\
A_{i}^{*}=\frac{\chi_{i} e^{-\lambda_{i} \theta_{i}} \omega_{i} U_{i}^{*} V^{*}}{\beta_{i}}+\frac{v_{i}\left(1-\chi_{i}\right) e^{-\tau_{i} \eta_{i}} \omega_{i} U_{i}^{*} V^{*}}{\beta_{i}\left(\zeta_{i}+v_{i}\right)}, & \varepsilon V^{*}=\mu B^{*}+\vartheta V^{*} B^{*},
\end{array}
$$

into the last equation of $\frac{d M_{1}}{d t}$, we get

$$
\begin{aligned}
& \frac{d M_{1}}{d t}=\sum_{i=1}^{n} \frac{\psi_{i}+\phi_{i}}{\omega_{i}}\left(1-\frac{u_{i}^{*}}{u_{i}}\right)\left(\gamma_{i} u_{i}^{*}-\gamma_{i} u_{i}\right) \\
& +\sum_{i=1}^{n} \psi_{i} u_{i}^{*} V^{*}\left[3-\frac{u_{i}^{*}}{u_{i}}+\frac{V}{V^{*}}-\frac{u_{i}\left(t-\tau_{i}\right) V\left(t-\tau_{i}\right) L_{i}^{*}}{u_{i}^{*} V^{*} L_{i}}\right. \\
& \left.-\frac{A_{i}^{*} L_{i}}{A_{i} L_{i}^{*}}-\frac{V^{*} A_{i}\left(t-\pi_{i}\right)}{V A_{i}^{*}}+\ln \left(\frac{U_{i}\left(t-\tau_{i}\right) V\left(t-\tau_{i}\right)}{U_{i} V}\right)+\ln \left(\frac{A_{i}\left(t-\pi_{i}\right)}{A_{i}}\right)\right] \\
& +\sum_{i=1}^{n} \phi_{i} u_{i}^{*} V^{*}\left[2-\frac{u_{i}^{*}}{u_{i}}+\frac{V}{V^{*}}-\frac{u_{i}\left(t-\lambda_{i}\right) V\left(t-\lambda_{i}\right) A_{i}^{*}}{u_{i}^{*} V^{*} A_{i}}\right. \\
& \left.-\frac{V^{*} A_{i}\left(t-\pi_{i}\right)}{V A_{i}^{*}}+\ln \left(\frac{U_{i}\left(t-\lambda_{i}\right) V\left(t-\lambda_{i}\right)}{U_{i} V}\right)+\ln \left(\frac{A_{i}\left(t-\pi_{i}\right)}{A_{i}}\right)\right] \\
& -\xi\left(V-V^{*}\right)-\rho\left(V-V^{*}\right) B+\rho\left(V-V^{*}\right) B^{*}-\rho\left(V-V^{*}\right) B^{*} \\
& +\frac{\rho\left(B-B^{*}\right)}{\varepsilon-\vartheta B^{*}}\left(\varepsilon V-\mu B-\vartheta V B-\varepsilon V^{*}+\mu B^{*}+\vartheta V^{*} B^{*}+\vartheta V B^{*}-\vartheta V B^{*}\right) \\
& =-\sum_{i=1}^{n} \frac{\gamma_{i}\left(\psi_{i}+\phi_{i}\right)\left(u_{i}-u_{i}^{*}\right)^{2}}{\omega_{i} U_{i}}+\sum_{i=1}^{n} \psi_{i} u_{i}^{*} V^{*}\left[3-\frac{u_{i}^{*}}{u_{i}}+\frac{V}{V^{*}}-\frac{U_{i}\left(t-\tau_{i}\right) V\left(t-\tau_{i}\right) L_{i}^{*}}{u_{i}^{*} V^{*} L_{i}}\right. \\
& \left.-\frac{A_{i}^{*} L_{i}}{A_{i} L_{i}^{*}}-\frac{V^{*} A_{i}\left(t-\pi_{i}\right)}{V A_{i}^{*}}+\ln \left(\frac{U_{i}\left(t-\tau_{i}\right) V\left(t-\tau_{i}\right)}{u_{i} V}\right)+\ln \left(\frac{A_{i}\left(t-\pi_{i}\right)}{A_{i}}\right)\right] \\
& +\sum_{i=1}^{n} \phi_{i} u_{i}^{*} V^{*}\left[2-\frac{u_{i}^{*}}{u_{i}}+\frac{V}{V^{*}}-\frac{u_{i}\left(t-\lambda_{i}\right) V\left(t-\lambda_{i}\right) A_{i}^{*}}{u_{i}^{*} V^{*} A_{i}}\right. \\
& \left.-\frac{V^{*} A_{i}\left(t-\pi_{i}\right)}{V A_{i}^{*}}+\ln \left(\frac{U_{i}\left(t-\lambda_{i}\right) V\left(t-\lambda_{i}\right)}{U_{i} V}\right)+\ln \left(\frac{A_{i}\left(t-\pi_{i}\right)}{A_{i}}\right)\right] \\
& -\left(\xi+\rho B^{*}\right)\left(V-V^{*}\right)-\rho\left(V-V^{*}\right)\left(B-B^{*}\right)+\frac{\rho\left(\varepsilon-\vartheta B^{*}\right)}{\left(\varepsilon-\vartheta B^{*}\right)}\left(B-B^{*}\right)\left(V-V^{*}\right)-\frac{\rho(\mu+\vartheta V)}{\left(\varepsilon-\vartheta B^{*}\right)}\left(B-B^{*}\right)^{2} .
\end{aligned}
$$


Note that

$$
-\left(\xi+\rho B^{*}\right)\left(V-V^{*}\right)=\sum_{i=1}^{n}\left(\psi_{i}+\phi_{i}\right) \mathrm{U}_{i}^{*} \mathrm{~V}^{*}\left(1-\frac{\mathrm{V}}{\mathrm{V}^{*}}\right)
$$

In addition, we have

$$
\begin{aligned}
\ln \left(\frac{U_{i}\left(t-\tau_{i}\right) V\left(t-\tau_{i}\right)}{U_{i} V}\right) & =\ln \left(\frac{U_{i}^{*}}{U_{i}}\right)+\ln \left(\frac{U_{i}\left(t-\tau_{i}\right) V\left(t-\tau_{i}\right) L_{i}^{*}}{U_{i}^{*} V^{*} L_{i}}\right)+\ln \left(\frac{A_{i}^{*} L_{i}}{A_{i} L_{i}^{*}}\right)+\ln \left(\frac{A_{i} V^{*}}{A_{i}^{*} V}\right), \\
\ln \left(\frac{U_{i}\left(t-\lambda_{i}\right) V\left(t-\lambda_{i}\right)}{U_{i} V}\right) & =\ln \left(\frac{U_{i}^{*}}{U_{i}}\right)+\ln \left(\frac{U_{i}\left(t-\lambda_{i}\right) V\left(t-\lambda_{i}\right) A_{i}^{*}}{U_{i}^{*} V^{*} A_{i}}\right)+\ln \left(\frac{A_{i} V^{*}}{A_{i}^{*} V}\right), \\
\ln \left(\frac{A_{i}\left(t-\pi_{i}\right)}{A_{i}}\right) & =\ln \left(\frac{V^{*} A_{i}\left(t-\pi_{i}\right)}{V A_{i}^{*}}\right)+\ln \left(\frac{A_{i}^{*} V}{A_{i} V^{*}}\right) .
\end{aligned}
$$

Simplifying the $\frac{d M_{1}}{d t}$ equation, we obtain

$$
\begin{aligned}
\frac{d M_{1}}{d t}= & -\sum_{i=1}^{n} \frac{\gamma_{i}\left(\psi_{i}+\phi_{i}\right)\left(U_{i}-U_{i}^{*}\right)^{2}}{\omega_{i} U_{i}}-\frac{\rho(\mu+\vartheta V)}{\varepsilon-\vartheta B^{*}}\left(B-B^{*}\right)^{2} \\
& -\sum_{i=1}^{n} \psi_{i} U_{i}^{*} V^{*}\left[G\left(\frac{U_{i}^{*}}{U_{i}}\right)+G\left(\frac{U_{i}\left(t-\tau_{i}\right) V\left(t-\tau_{i}\right) L_{i}^{*}}{U_{i}^{*} V^{*} L_{i}}\right)+G\left(\frac{A_{i}^{*} L_{i}}{A_{i} L_{i}^{*}}\right)+G\left(\frac{V^{*} A_{i}\left(t-\pi_{i}\right)}{V A_{i}^{*}}\right)\right] \\
& -\sum_{i=1}^{n} \phi_{i} U_{i}^{*} V^{*}\left[G\left(\frac{U_{i}^{*}}{U_{i}}\right)+G\left(\frac{U_{i}\left(t-\lambda_{i}\right) V\left(t-\lambda_{i}\right) A_{i}^{*}}{U_{i}^{*} V^{*} A_{i}}\right)+G\left(\frac{V^{*} A_{i}\left(t-\pi_{i}\right)}{V A_{i}^{*}}\right)\right] .
\end{aligned}
$$

Therefore, $\frac{d M_{1}}{d t} \leqslant 0$ for all $U_{i}, L_{i}, A_{i}, V, B>0$. In addition, $\frac{d M_{1}}{d t}=0$ if $U_{i}=U_{i}^{*}, L_{i}=L_{i}^{*}, A_{i}=A_{i}^{*}$, $\mathrm{V}=\mathrm{V}^{*}$ and $\mathrm{B}=\mathrm{B}^{*}$. In the same way as the proof of Theorem 4.1 we get that the largest invariance subset contains a single point $E^{*}$. Applying L.I.P, we obtain that $E^{*}$ is G.A.S if $R_{0}>0$.

\section{Example and numerical simulations}

In the following, we carry out a special case of model (2.1) and perform some numerical simulations to illustrate the theoretical results and to investigate the effect of some parameters. It was reported in [41] that HIV infects two types of immune system cells: $C D 4^{+} \mathrm{T}$ cells and macrophages, i.e., $\mathrm{n}=2$. Let us introduce an HIV infection model under the effect of highly active anti-retroviral therapy (HAART) consisting of a combination of reverse transcriptase inhibitor (RTI) and protease inhibitor (PI):

$$
\begin{aligned}
\dot{\mathrm{U}}_{1} & =\rho_{1}-\gamma_{1} \mathrm{U}_{1}-\left(1-\epsilon_{\mathrm{r}_{1}}\right) \omega_{1} \mathrm{U}_{1} \mathrm{~V}, \\
\dot{\mathrm{U}}_{2} & =\rho_{2}-\gamma_{2} \mathrm{U}_{2}-\left(1-\epsilon_{\mathrm{r}_{2}}\right) \omega_{2} \mathrm{U}_{2} \mathrm{~V}, \\
\dot{\mathrm{L}_{1}} & =\left(1-\epsilon_{\mathrm{r}_{1}}\right)\left(1-\chi_{1}\right) e^{-\tau_{1} \eta_{1}} \omega_{1} \mathrm{U}_{1}\left(\mathrm{t}-\tau_{1}\right) \mathrm{V}\left(\mathrm{t}-\tau_{1}\right)-\left(\zeta_{1}+v_{1}\right) \mathrm{L}_{1}, \\
\dot{\mathrm{L}_{2}} & =\left(1-\epsilon_{\mathrm{r}_{2}}\right)\left(1-\chi_{2}\right) e^{-\tau_{2} \eta_{2}} \omega_{2} \mathrm{U}_{2}\left(\mathrm{t}-\tau_{2}\right) \mathrm{V}\left(\mathrm{t}-\tau_{2}\right)-\left(\zeta_{2}+v_{2}\right) \mathrm{L}_{2}, \\
\dot{A_{1}} & =\left(1-\epsilon_{\mathrm{r}_{1}}\right) \chi_{1} e^{-\lambda_{1} \theta_{1}} \omega_{1} \mathrm{U}_{1}\left(\mathrm{t}-\lambda_{1}\right) \mathrm{V}\left(\mathrm{t}-\lambda_{1}\right)+v_{1} \mathrm{~L}_{1}-\beta_{1} A_{1}, \\
\dot{A_{2}} & =\left(1-\epsilon_{\mathrm{r}_{2}}\right) \chi_{2} e^{-\lambda_{2} \theta_{2}} \omega_{2} \mathrm{U}_{2}\left(\mathrm{t}-\lambda_{2}\right) \mathrm{V}\left(\mathrm{t}-\lambda_{2}\right)+v_{2} \mathrm{~L}_{2}-\beta_{2} A_{2}, \\
\dot{\mathrm{V}} & =\left(1-\epsilon_{\mathrm{p}_{1}}\right) \varkappa_{1} e^{-\pi_{1} \iota_{1}} \mathrm{~A}_{1}\left(\mathrm{t}-\pi_{1}\right)+\left(1-\epsilon_{\mathrm{p}_{2}}\right) \varkappa_{2} e^{-\pi_{2} \iota_{2}} \mathrm{~A}_{2}\left(\mathrm{t}-\pi_{2}\right)-\xi \mathrm{V}-\rho \mathrm{VB}, \\
\dot{\mathrm{B}} & =\varepsilon V-\mu \mathrm{V}-\vartheta \mathrm{VB},
\end{aligned}
$$

where $\epsilon_{r_{i}} \in[0,1]$ is the efficacy of the RTI drugs, while $\epsilon_{p_{i}} \in[0,1]$ is the efficacy of the PI drugs. Consequently, the parameter $R_{0}$ of model (5.1) is given by:

$$
R_{0}=\sum_{i=1}^{2} \frac{\varkappa_{i} \omega_{i} \rho_{i}\left(1-\epsilon_{p_{i}}\right)\left(1-\epsilon_{r_{i}}\right)\left(\chi_{i}\left(\zeta_{i}+\nu_{i}\right) e^{-\lambda_{i} \theta_{i}}+v_{i}\left(1-\chi_{i}\right) e^{-\tau_{i} \eta_{i}}\right) e^{-\pi_{i} \iota_{i}}}{\xi \beta_{i} \gamma_{i}\left(\zeta_{i}+\nu_{i}\right)}
$$


We perform some numerical simulation for model (5.1) using the values of parameters given in Table 1. All the computations will be carried out by MATLAB. We have chosen the values of parameters of the model to perform the numerical simulations.

\subsection{Stability of equilibria}

In this case, we want to show that the theoretical results of Theorems 1-2 are consistent with the numerical simulations. Thus, we consider three different initial conditions as follows:

IC1: $\mathrm{U}_{\mathrm{i}}(\mathrm{s})=300, \mathrm{~L}_{\mathrm{i}}(\mathrm{s})=10, \mathrm{~A}_{\mathrm{i}}(\mathrm{s})=30, \mathrm{~V}(\mathrm{~s})=8, \mathrm{~B}(\mathrm{~s})=25$;

IC2: $U_{i}(s)=400, L_{i}(s)=7, A_{i}(s)=20, V(s)=5, B(s)=20$;

IC3: $\mathrm{U}_{\mathrm{i}}(\mathrm{s})=600, \mathrm{~L}_{\mathrm{i}}(\mathrm{s})=3, \mathrm{~A}_{\mathrm{i}}(\mathrm{s})=10, \mathrm{~V}(\mathrm{~s})=3, \mathrm{~B}(\mathrm{~s})=15$,

where $s \in[-\tilde{T}, 0]$ and $\tilde{T}=\max \left\{\tau_{1}, \tau_{2}, \lambda_{1}, \lambda_{2}, \pi_{1}, \pi_{2}\right\}$.

We figure out the following:

(i) when $\omega_{i}=5 \times 10^{-5}$, then $R_{0}=0.609164<1$ which means that by Theorem 4.1 the trajectories of solution tend to the infection-free equilibrium $E^{0}$ (see Figure 1). From the biological viewpoint, the HIV is cleared from patient's body;

(ii) when $\omega_{i}=5 \times 10^{-4}$, then $R_{0}=6.09164>1$. According to Theorem 4.2 , the trajectories of solution head for the chronic-infection equilibrium $E^{*}=(486.7,469.5,4.885,3.679,16.07,9.638,30.16,19.36)$ (see Figure 1). In this case the HIV is deep-seated in patient's plasma.

As we can see from Figure 1, the outcomes are agreed with the theoretical results. The values of parameters we take in this case are listed in Table 1 with the following values of the rest of parameters: $\tau_{1}=0.05, \tau_{2}=0.01, \lambda_{1}=0.2, \lambda_{2}=0.08, \pi_{1}=0.05, \pi_{2}=0.02, \epsilon_{\mathrm{r}_{1}}=0.3, \epsilon_{\mathrm{r}_{2}}=0.25, \epsilon_{\mathrm{p}_{1}}=0.15, \epsilon_{\mathrm{p}_{2}}=0.17$, and $\vartheta=0.01$.

\subsection{The effect of B cells impairment parameter}

Since the main contribution of our model is to incorporate the impaired B cells, so we want to study its impact on the virus dynamics. We consider different values of $\vartheta$ to see what will happen to other components. Figure 2 indicates that there is a positive correlation between the value of impairment parameter and the amount of HIV particles. In other words, the HIV particles are increased as $\vartheta$ is increased which leads to more infection of uninfected cells and then increasing of concentrations of all types of infected cells. When the uninfected cells' concentrations are too low, then the patient is said to have acquired immunodeficiency syndrome (AIDS). Therefore, the impaired B cells will help HIV particles to reach AIDS stage more rapidly. The data we take in this case are listed in Table 1 with the following values of the rest of parameters: $\tau_{1}=0.05, \tau_{2}=0.01, \lambda_{1}=0.2, \lambda_{2}=0.08, \pi_{1}=0.05, \pi_{2}=0.02$, $\epsilon_{\mathrm{r}_{1}}=0.3, \epsilon_{\mathrm{r}_{2}}=0.25, \epsilon_{\mathrm{p}_{1}}=0.15, \epsilon_{\mathrm{p}_{2}}=0.17$ and $\omega_{\mathrm{i}}=0.001 ; i=1,2$.

\subsection{The effect of HAART}

Let $\epsilon=\epsilon_{r_{i}}=\epsilon_{p_{i}}, i=1,2$ to examine what is the value of $\epsilon$ that makes $R_{0}=1$ for model (5.1). In this case, we use the values of parameters listed in Table 1 with the following values of the rest of parameters $\tau_{1}=0.05, \tau_{2}=0.01, \lambda_{1}=0.2, \lambda_{2}=0.08, \pi_{1}=0.05, \pi_{2}=0.02, \vartheta=0.01$, and $\omega_{i}=0.001 ; i=1,2$ into Eq. (5.2) then we get $\epsilon^{\text {critical }}=0.777664$, i.e., we have two situations:

(i) if $0<\epsilon<0.777664$, then $R_{0}>1$ and HIV particles are persistent;

(ii) if $0.777664<\epsilon \leqslant 1$, then $R_{0}<1$ and the patient is healed.

Moreover, the behaviours of the variables with different values of efficiencies of drugs are presented in Figure 3. We employ the following data:

group (1): $\epsilon_{\mathrm{r}_{1}}=0.04, \epsilon_{\mathrm{r}_{1}}=0.03, \epsilon_{\mathrm{p}_{1}}=0.05$, and $\epsilon_{\mathrm{p}_{2}}=0.07$.

group (2): $\epsilon_{\mathrm{r}_{1}}=0.3, \epsilon_{\mathrm{r}_{1}}=0.1, \epsilon_{\mathrm{p}_{1}}=0.5$, and $\epsilon_{\mathrm{p}_{2}}=0.6$. 
group (3): $\epsilon_{\mathrm{r}_{1}}=0.4, \epsilon_{\mathrm{r}_{1}}=0.5, \epsilon_{\mathrm{p}_{1}}=0.7$, and $\epsilon_{\mathrm{p}_{2}}=0.6$.

group (4): $\epsilon_{\mathrm{r}_{1}}=0.8, \epsilon_{\mathrm{r}_{1}}=0.8, \epsilon_{\mathrm{p}_{1}}=0.8$, and $\epsilon_{\mathrm{p}_{2}}=0.8$.

From the figure, we can see that the increasing of the efficacy of drugs will increase the concentration of the uninfected cells and decrease the concentrations of latent infected cells, active infected cells, HIV particles and B cells.

\subsection{The effect of time delays parameters}

Figure 4 depicts the effect of time delays on the virus dynamics. We consider these values of time delays:

set (1): $\tau_{1}=0.5, \tau_{2}=0.2, \lambda_{1}=0.9, \lambda_{2}=0.7, \pi_{1}=0.5$, and $\pi_{2}=0.4$.

set (2): $\tau_{1}=0.7, \tau_{2}=0.4, \lambda_{1}=1, \lambda_{2}=0.9, \pi_{1}=0.8$, and $\pi_{2}=0.6$.

set (3): $\tau_{1}=0.9, \tau_{2}=0.6, \lambda_{1}=1.2, \lambda_{2}=1.1, \pi_{1}=1.1$, and $\pi_{2}=0.8$.

set (4): $\tau_{1}=1.4, \tau_{2}=1.4, \lambda_{1}=1.4, \lambda_{2}=1.4, \pi_{1}=1.4$, and $\pi_{2}=1.4$.

It is observed that the effect of time delays on the dynamical behavior of the HIV is similar as the effect of HAART. So it may help to develop a new class of treatment to enlarge the delay period and then suppress the HIV replication. In addition, we let $S=\tau_{i}=\lambda_{i}=\pi_{i} ; i=1,2$ and solve Eq. (5.2) using the values of parameter given in Table 1 with the following values of the rest of parameters $\epsilon_{\mathrm{r}_{1}}=0.3$, $\epsilon_{\mathrm{r}_{2}}=0.25, \epsilon_{\mathrm{p}_{1}}=0.15, \epsilon_{\mathrm{p}_{2}}=0.17, \vartheta=0.01$ and $\omega_{i}=0.001 ; i=1,2$ to find the critical value of time delays that make $\mathrm{R}_{0}=1$. We obtain $\mathrm{S}^{\text {critical }}=1.32035$. When $0<\mathrm{S}<1.32035$, the trajectories tend to $\mathrm{E}^{*}$ while, if $S>1.32035$, the trajectories tend towards $E^{0}$ and the body is cleared from HIV particles.

\section{Conclusion and discussion}

The intention of this paper was to incorporate the effect of impairment of B cell functions into the virus infection model with multi-target cells. We have introduced a virus dynamics model taking into account multi-target cells, latent stage of infection, three different types of time delays and impairment of B cell response. We have found out that the model is biologically acceptable in the sense that all solutions are nonnegative and bounded. We have demonstrated the basic reproduction number $R_{0}$ which determined the existence and global stability of equilibria. We have proved that the infection-free equilibrium $E^{0}$ always exists, while an endemic-infection equilibrium $E^{*}$ exists only if $R_{0}>1$. We have constructed proper Lyapunov functionals and then applied LaSalle's invariance principle to show that if $R_{0}<1$, then $E^{0}$ is G.A.S, on the other hand, if $R_{0}>1$, the other equilibrium $E^{*}$ is G.A.S. The basic reproduction number $R_{0}$ of model (2.1) is given as $R_{0}=\sum_{i=1}^{n} R_{0 i}>R_{01}$, where $R_{01}$ is the basic reproduction number of HIV model with one class of target cells. Therefore, neglecting multi-target cells will lead to under-estimated basic reproduction number.

We have set $n=2$ in model (2.1) to generate an HIV model with two types of target cells, CD4 ${ }^{+}$T cells and macrophages. We have combined HAART into the proposed model and showed that the theoretical results are convenient with the visual results. The effects of impairment of B cells functions, time delays and HAART treatment on the virus dynamics were addressed. We conclude that

- the time delays play the same influence of HAART treatment in stabilizing the system around the infection-free equilibrium;

- when the B cells lose their functions during the viral infection, the amount of antibodies produced by the B cells decreases and then the amount of HIV particles increases. Therefore, HAART treatment is needed to improve the health of the infected patient. 
For comparison purposes, we consider the model (5.1) by neglecting the immune impairment, i.e., $\vartheta=0$ as:

$$
\begin{aligned}
\dot{\mathrm{U}}_{1} & =\rho_{1}-\gamma_{1} \mathrm{U}_{1}-\left(1-\epsilon_{\mathrm{r}_{1}}\right) \omega_{1} \mathrm{U}_{1} \mathrm{~V}, \\
\dot{\mathrm{U}}_{2} & =\rho_{2}-\gamma_{2} \mathrm{U}_{2}-\left(1-\epsilon_{\mathrm{r}_{2}}\right) \omega_{2} \mathrm{U}_{2} \mathrm{~V}, \\
\dot{\mathrm{L}_{1}} & =\left(1-\epsilon_{\mathrm{r}_{1}}\right)\left(1-\chi_{1}\right) e^{-\tau_{1} \eta_{1}} \omega_{1} \mathrm{U}_{1}\left(\mathrm{t}-\tau_{1}\right) \mathrm{V}\left(\mathrm{t}-\tau_{1}\right)-\left(\zeta_{1}+v_{1}\right) \mathrm{L}_{1}, \\
\dot{\mathrm{L}_{2}} & =\left(1-\epsilon_{\mathrm{r}_{2}}\right)\left(1-\chi_{2}\right) e^{-\tau_{2} \eta_{2}} \omega_{2} \mathrm{U}_{2}\left(\mathrm{t}-\tau_{2}\right) \mathrm{V}\left(\mathrm{t}-\tau_{2}\right)-\left(\zeta_{2}+v_{2}\right) \mathrm{L}_{2}, \\
\dot{\mathrm{A}_{1}} & =\left(1-\epsilon_{\mathrm{r}_{1}}\right) \chi_{1} e^{-\lambda_{1} \theta_{1}} \omega_{1} \mathrm{U}_{1}\left(\mathrm{t}-\lambda_{1}\right) \mathrm{V}\left(\mathrm{t}-\lambda_{1}\right)+v_{1} \mathrm{~L}_{1}-\beta_{1} A_{1}, \\
\dot{\mathrm{A}_{2}} & =\left(1-\epsilon_{\mathrm{r}_{2}}\right) \chi_{2} e^{-\lambda_{2} \theta_{2}} \omega_{2} \mathrm{U}_{2}\left(\mathrm{t}-\lambda_{2}\right) \mathrm{V}\left(\mathrm{t}-\lambda_{2}\right)+v_{2} \mathrm{~L}_{2}-\beta_{2} A_{2}, \\
\dot{\mathrm{V}} & =\left(1-\epsilon_{\mathrm{p}_{1}}\right) \varkappa_{1} e^{-\pi_{1} \iota_{1}} \mathrm{~A}_{1}\left(\mathrm{t}-\pi_{1}\right)+\left(1-\epsilon_{\mathrm{p}_{2}}\right) \varkappa_{2} e^{-\pi_{2} \iota_{2}} A_{2}\left(\mathrm{t}-\pi_{2}\right)-\xi \mathrm{V}-\rho \mathrm{VB}, \\
\dot{\mathrm{B}} & =\varepsilon \mathrm{V}-\mu \mathrm{BB} .
\end{aligned}
$$

Let the endemic-infection equilibrium thought as a function of $\epsilon=\epsilon_{r_{i}}=\epsilon_{p_{i}}, i=1,2$ be given as: $E^{*}(\epsilon)=\left(u_{1}^{*}(\epsilon), U_{2}^{*}(\epsilon), L_{1}^{*}(\epsilon), L_{2}^{*}(\epsilon), A_{1}^{*}(\epsilon), A_{2}^{*}(\epsilon), V^{*}(\epsilon), B^{*}(\epsilon)\right)$. Then the objective is to design treatment with efficacy $\epsilon$ to keep the concentration of the HIV particles below a given level, i.e.,

$$
0 \leqslant V^{*}(\epsilon) \leqslant \bar{V}, \text { for all } \epsilon^{*} \leqslant \epsilon \leqslant \epsilon^{* *} .
$$

Let us use the value of parameters in Table 1 and the values of parameters given in Case $5.3, \bar{V}=0.2$ and $\vartheta$ is varied. We calculate $\epsilon^{*}$ and $\epsilon^{* *}$ for two models.

(I) Model (6.1) (i.e., ignoring the impairment of B cells): we find that the inequality (6.2) is satisfied when $0.782704 \leqslant \epsilon \leqslant 0.816749$.

(II) Model (5.1) (i.e., incorporating the impairment of B cells): we take $\vartheta=0.4$ and find that the inequality (6.2) is satisfied when $0.81237 \leqslant \epsilon \leqslant 0.816749$.

Therefore, if we apply drug with efficacy $\epsilon$ such that $0.782704 \leqslant \epsilon \leqslant 0.81237$, this guarantees that $\mathrm{V}^{*}(\epsilon) \leqslant 0.2$ for model (6.1), but $\mathrm{V}^{*}(\epsilon)>0.2$ for model (5.1). Therefore, a more accurate drug efficacy required to reduce the concentration of the virus particle to a lower value is calculated by using our model. This shows the importance of considering the effect of immune impairment in the virus dynamics models.

Table 1: Values of fixed parameters.

\begin{tabular}{|c|c|c|c|}
\hline Parameter & Value & Parameter & Value \\
\hline$\rho_{i}$ & 10 & $\eta_{i}$ & 1 \\
$\gamma_{i}$ & 0.01 & $\theta_{i}$ & 1 \\
$\chi_{1}$ & 0.3 & $\iota_{i}$ & 1 \\
$\chi_{2}$ & 0.44 & $\nu_{1}$ & 0.4 \\
$\zeta_{1}$ & 0.3 & $\gamma_{2}$ & 0.2 \\
$\zeta_{2}$ & 0.6 & $\beta_{1}$ & 0.2 \\
$\varkappa_{1}$ & 5 & $\beta_{2}$ & 0.3 \\
$\varkappa_{2}$ & 3 & $\xi$ & 1 \\
$\rho$ & 0.1 & $\varepsilon$ & 0.2 \\
$\mu$ & 0.01 & & \\
\hline
\end{tabular}

We noticed that our proposed virus dynamics model is developed with one mode of infection namely virus-to-cell infection. Therefore our model can be extended to incorporate cell-to-cell transmission mode where the infected cells could directly infect the adjacent uninfected target cells [8,22, 27]. We mentioned that our virus infection model assumed that the incidence rate is given by bilinear form. However, when the concentration of virus in plasma is high, the bilinear form cannot characterize the virus dynamics accurately [20,33]. Our proposed model can be generalized to combine another types of incidence rates such as saturated, Holling type-II, Beddington-DeAngelis, Crowley-Martin and general incidence rate. In addition, the effect of diffusion $[2,3,15,16]$ as well as stochastic interactions [29] were neglected in our proposed model. However, it is more practical to improve our model to include these natural phenomena. 

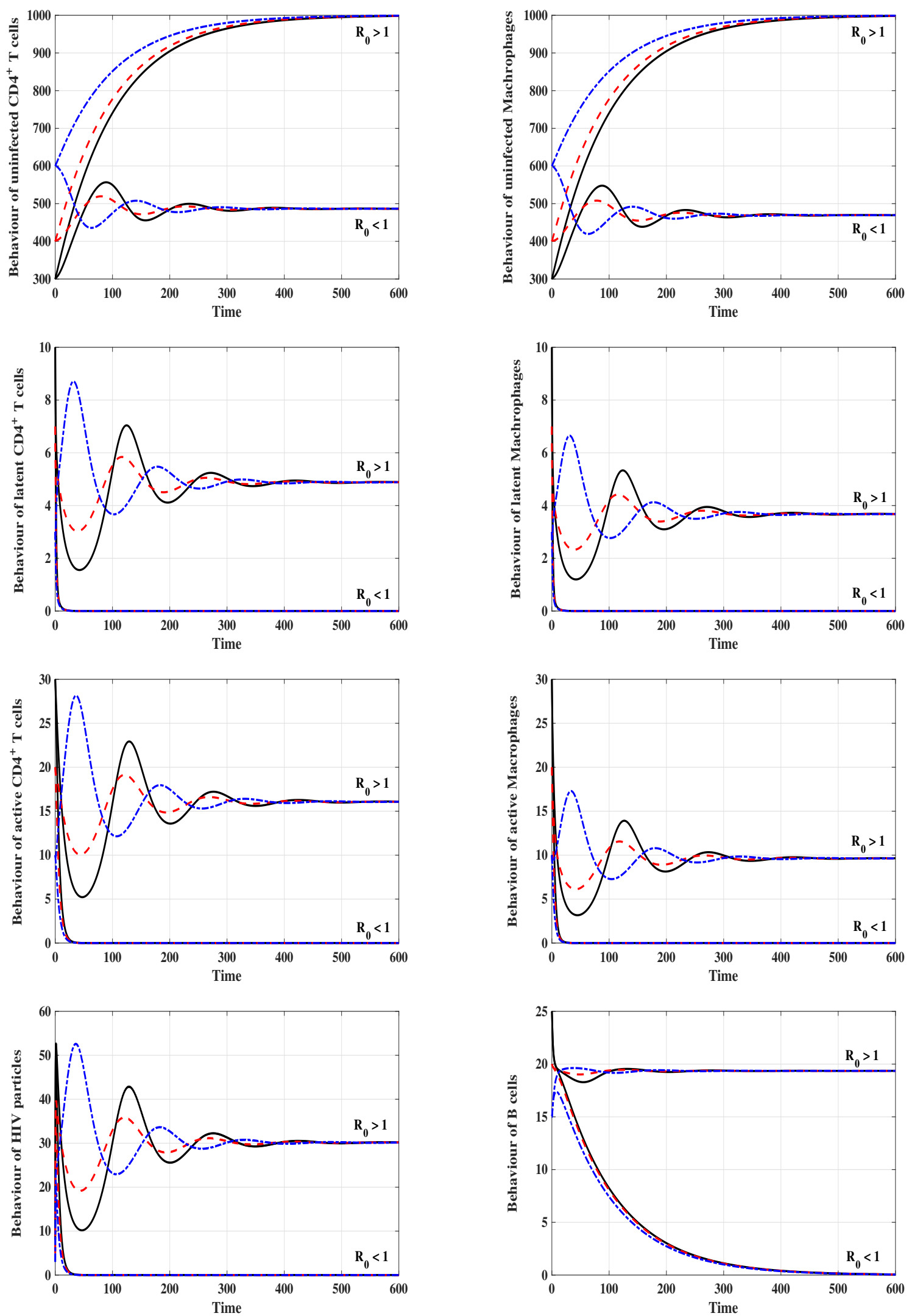

Figure 1: Solution trajectories when $\mathcal{R}_{0}<1$ and $\mathcal{R}_{0}>1$ with different initial conditions. 

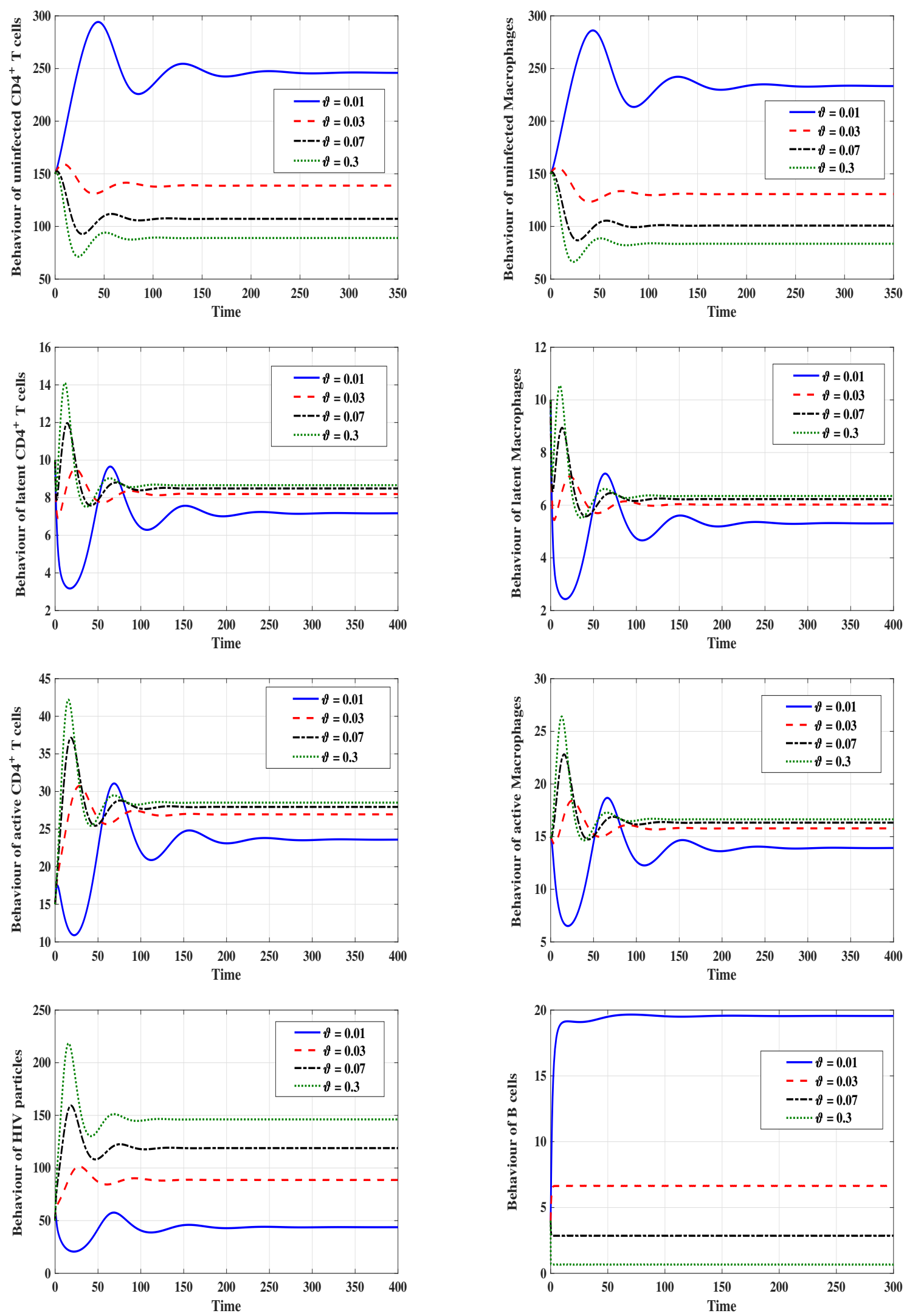

Figure 2: Solution trajectories for different values of $\vartheta$. 

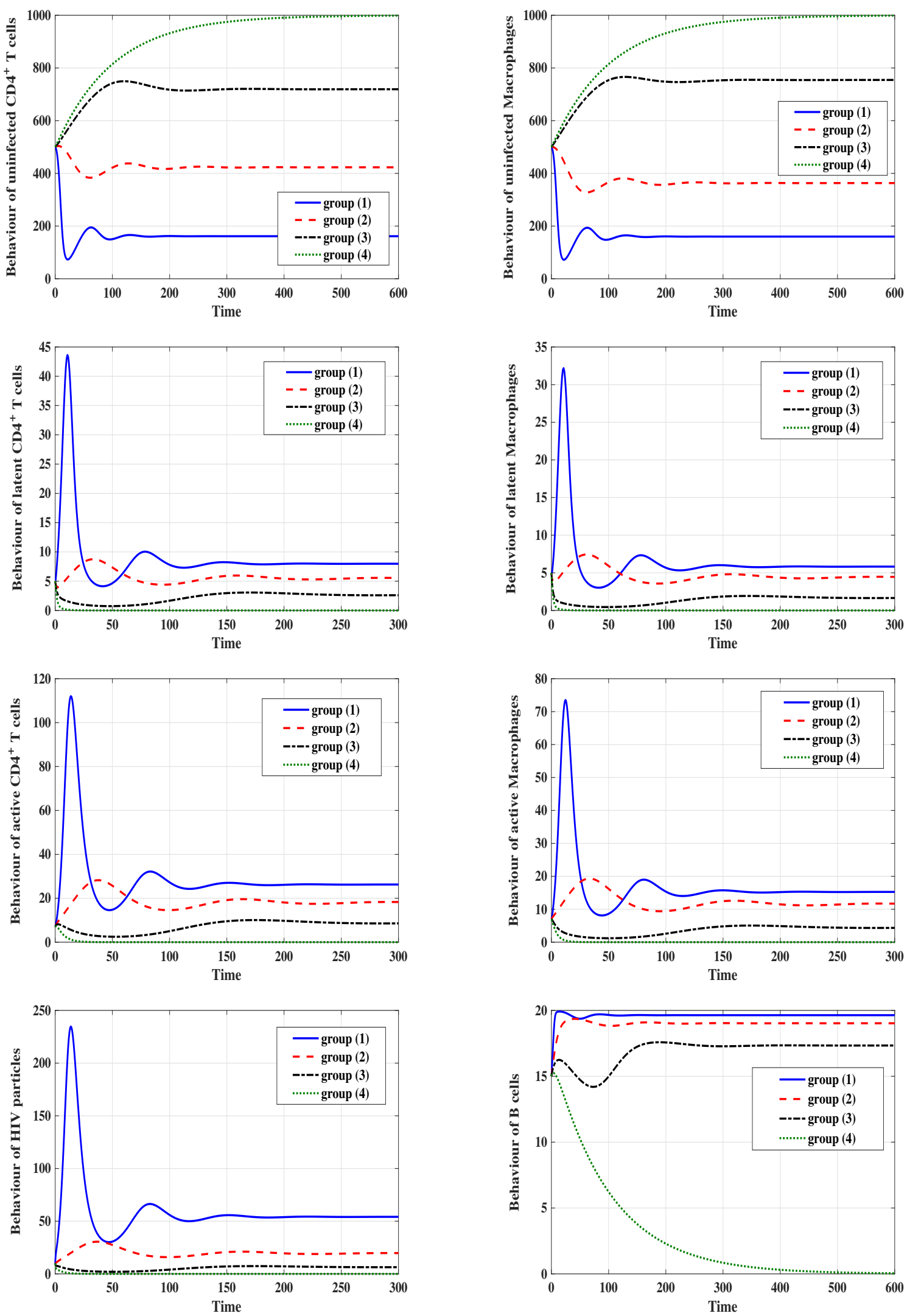

Figure 3: Solution trajectories for different values of the efficacy of PI and RTI drugs. 

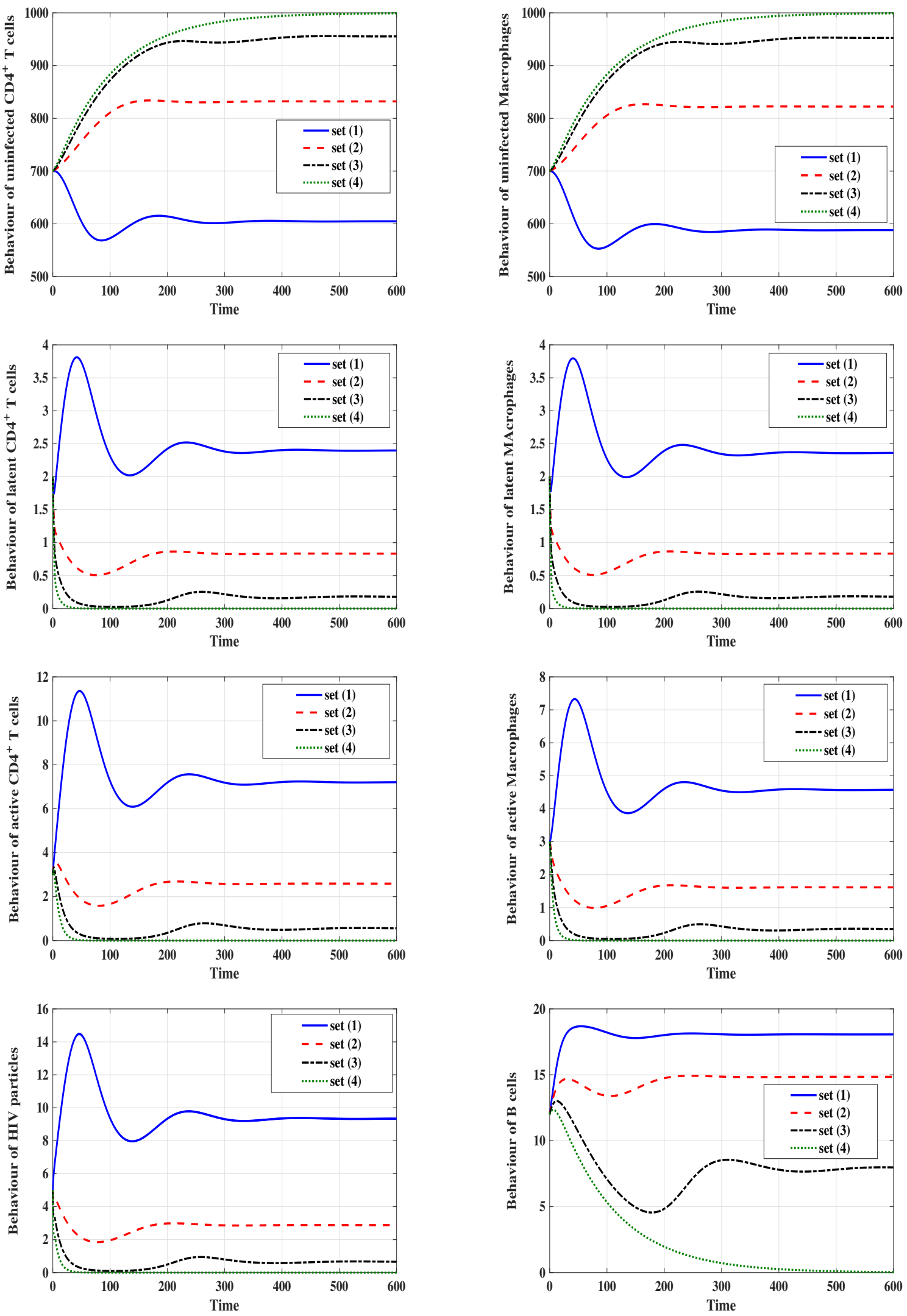

Figure 4: Solution trajectories for different values of time delays. 


\section{References}

[1] S. Amu, N. Ruffin, B. Rethi, F. Chiodi, Impairment of B-cell functions during HIV-1 infection, AIDS, 27 (2013), $2323-$ 2334. 1

[2] N. Bellomo, K. J. Painter, Y. Tao, M. Winkler, Occurrence vs. Absence of taxis-driven instabilities in a May-Nowak model for virus infection, SIAM J. Appl. Math., 79 (2019), 1990-2010. 6

[3] N. Bellomo, Y. Tao, Stabilization in a chemotaxis model for virus infection, Discrete. Cont. Dyn Syst.-S, 13 (2020), 105-117. 6

[4] C. Caglioti, E. Lalle, C. Castilletti, F. Carletti, M. R. Capobianchi, L. Bordi, Chikungunya virus infection: an overview, New Microbiol., 36 (2013), 211-227. 1

[5] D. S. Callaway, A. S. Perelson, HIV-1 infection and low steady state viral loads, Bull. Math. Biol., 64 (2002), 29-64. 1

[6] F. Chiodi, G. Scarlatti, HIV-Induced damage of B cells and production of HIV neutralizing antibodies, Front. Immunol., 9 (2018), 4 pages. 1

[7] R. V. Culshaw, S. Ruan, A delay-differential equation model of HIV infection of CD4 ${ }^{+}$T-cells, Math. Biosci., 165 (2000), 27-39. 1

[8] R. V. Culshaw, S. Ruan, G. Webb, A mathematical model of cell-to-cell spread of HIV-1 that includes a time delay, J. Math. Biol., 46 (2003), 425-444. 6

[9] A. De Milito, B lymphocyte dysfunctions in HIV Infection, Curr. HIV. Res., 2 (2004), 11-21. 1

[10] N. M. Dixit, M. Markowitz, D. D. Ho, A. S. Perelson, Estimates of intracellular delay and average drug efficacy from viral load data of HIV-infected individuals under antiretroviral therapy, Antivir. Ther., 9 (2004), 237-246. 1

[11] A. M. Elaiw, Global properties of a class of HIV models, Nonlinear Anal. Real World Appl., 11 (2010), 2253-2263. 1

[12] A. M. Elaiw, Global properties of a class of virus infection models with multi-target cells, Nonlinear Dynam., 69 (2012), 423-435. 1

[13] A. M. Elaiw, R. M. Abukwaik, E. O. Alzahrani, Global properties of a cell mediated immunity in HIV infection model with two classes of target cells and distributed delays, Int. J. Biomath., 7 (2014), 25 pages. 1

[14] A. M. Elaiw, T. O. Alade, S. A. Alsulami, Global stability of within-host virus dynamics models with multi-target cells, Mathematics, 6 (2018), 19 pages. 1

[15] A. M. Elaiw, A. D. AlAgha, Analysis of a delayed and diffusive oncolytic M1 virotherapy model with immune response, Nonlinear Anal. Real World Appl., 55 (2020), 32 pages. 6

[16] A. M. Elaiw, A. D. AlAgha, Global analysis of a reaction-diffusion within-host malaria infection model with adaptive immune response, Mathematics, 8 (2020), 32 pages. 6

[17] A. M. Elaiw, N. A. Almuallem, Global dynamics of delay-distributed HIV infection models with differential drug efficacy in cocirculating target cells, Math. Methods Appl. Sci., 39 (2016), 4-31. 1

[18] A. M. Elaiw, M. A. Alshaikh, Stability of discrete-time HIV dynamics models with three categories of infected CD4 ${ }^{+}$ T-cells, Adv. Difference Equ., 2019 (2019), 24 pages. 1

[19] A. M. Elaiw, M. A. Alshaikh, Stability of a discrete-time general delayed viral model with antibody and cell-mediated immune responses, Adv. Difference Equ., 2020 (2020), 1-51. 1

[20] A. M. Elaiw, N. H. AlShamrani, Global stability of a delayed adaptive immunity viral infection with two routes of infection and multi-stages of infected cells, Commun. Nonlinear Sci. Numer. Simul., 86 (2020), 27 pages. 6

[21] A. M. Elaiw, N. H. AlShamrani, Stability of a general CTL-mediated immunity HIV infection model with silent infected cell-to-cell spread, Adv. Difference Equ., 2020 (2020), 25 pages. 1

[22] A. M. Elaiw, S. F. Alshehaiween, Global stability of delay-distributed viral infection model with two modes of viral transmission and B-cell impairment, Math. Methods Appl. Sci., 43 (2020), 6677-6701. 6

[23] A. M. Elaiw, S. F. Alshehaiween, A. D. Hobiny, Global properties of a delay-distributed HIV dynamics model including impairment of B-cell functions, Mathematics, 7 (2019), 27 pages. 1

[24] A. M. Elaiw, E. K. Elnahary, Analysis of general humoral immunity HIV dynamics model with HAART and distributed delays, Mathematics, 7 (2019), 35 pages.

[25] A. M. Elaiw, E. K. Elnahary, A. A. Raezah, Effect of cellular reservoirs and delays on the global dynamics of HIV, Adv. Difference Equ., 2018 (2018), 36 pages.

[26] A. M. Elaiw, I. A. Hassanien, S. A. Azoz, Global stability of HIV infection models with intracellular delays, J. Korean Math. Soc., 49 (2012), 779-794. 1

[27] A. M. Elaiw, A. A. Raezah, Stability of general virus dynamics models with both cellular and viral infections and delays, Math. Methods Appl. Sci., 40 (2017), 5863-5880. 6

[28] N. Faried, M. S. S. Ali, H. H. Sakr, Fuzzy soft inner product spaces, Appl. Math. Inf. Sci., 14 (2020), 709-720. 1

[29] L. Gibelli, A. Elaiw, M. A. Alghamdi, A. M. Althiabi, Heterogeneous population dynamics of active particles: Progression, mutations, and selection dynamics, Math. Models Methods Appl. Sci., 27 (2017), 617-640. 6

[30] C. Gross, A. K. Thoma-Kress, Molecular Mechanisms of HTLV-1 Cell-to-Cell Transmission, Viruses, 8 (2016), 22 pages. 1

[31] J. K. Hale, S. M. Verduyn Lunel, Introduction to functional differential equations, Springer-Verlag, New York, (1993). 3

[32] A. Herz, S. Bonhoeffer, R. M. Anderson, R. M. May, M. A. Nowak, Viral dynamics in vivo: Limitations on estimates of intracellular delay and virus decay, Proc. Natl. Acad. Sci., 93 (1996), 7247-7251. 1 
[33] G. Huang, Y. Takeuchi, W. Ma, Lyapunov functionals for delay differential equations model of viral infections, SIAM J. Appl. Math., 70 (2010), 2693-2708. 6

[34] J. P. LaSalle, The Stability of Dynamical Systems, in: Regional Conference Series in Applied Mathematics, SIAM, Philadelaphia, (1976). 4

[35] M. Y. Li, L. C. Wang, Backward bifurcation in a mathematical model for HIV infection in vivo with anti-retroviral treatment, Nonlinear Anal. Real World Appl., 17 (2014), 147-160. 1

[36] P. Lydyard, A. Whelan, M. Fanger, BIOS Instant notes in immunology, Taylor \& Francis, London, (2005). 1

[37] P. W. Nelson, J. D. Murray, A. S. Perelson, A model of HIV-1 pathogenesis that includes an intracellular delay, Math. Biosci., 163 (2000), 201-215. 1

[38] M. A. Nowak, C. R. M. Bangham. Population dynamics of immune responses to persistent viruses, Science, 272 (1996), 74-79. 1

[39] M. A. Nowak, R. M. May, Virus dynamics: Mathematical Principles of Immunology and Virology, Oxford University Press, Oxford, (2000). 1

[40] S. Pankavich, The effects of latent infection on the dynamics of HIV, Differ. Equ. Dyn. Syst., 24 (2016), 281-303. 1

[41] A. S. Perelson, P. Essunger, Y. Cao, M. Vesanen, A. Hurley, K. Saksela, M. Markowitz, D. D. Ho, Decay characteristics of HIV-1 infected compartments during combination therapy, Nature, 387 (1997), 188-191. 1, 5

[42] P. K. Roy, A. N. Chatterjee, D. Greenhalgh, Q. J. A. Khan, Long term dynamics in a mathematical model of HIV-1 infection with delay in different variants of the basic drug therapy model, Nonlinear Anal. Real World Appl., 14 (2013), 1621-1633. 1

[43] L. M. Sompayrac, How the immune system works, Wiley-Blackwell, Hoboken, (2011). 1

[44] P. K. Srivastava, M. Banerjee, P. Chandra, Dynamical Model of in-host HIV infection: With Drug therapy and Multi viral strains, J. Biol. Syst., 20 (2012), 303-325. 1

[45] X. X. Tian, J. L. Wang, Stability analysis for viral infection model with multi-target cells, Beddington-DeAngelis functional response, and humoral immunity, Discrete Dyn. Nat. Soc., 2015 (2015), 11 pages. 1

[46] K. F. Wang, A. J. Fan, A. Torres, Global properties of an improved hepatitis B virus model, Nonlinear Anal. Real World Appl., 11 (2010), 3131-3138. 1

[47] J. J. Wang, J. L. Lang, F. Li, Constructing Lyapunov functionals for a delayed viral infection model with multi-target cells, nonlinear incidence rate, state-dependent removal rate, J. Nonlinear Sci. Appl., 9 (2016), 524-536. 1

[48] L. C. Wang, M. Y. Li, D. Kirschner, Mathematical analysis of the global dynamics of a model for HTLV-I infection and ATL progression, Math. Biosci., 179 (2002), 207-217. 1

[49] J. J. Wang, J. M. Pang, T. Kuniya, A note on global stability for malaria infections model with latencies, Math. Biosci. Eng., 11 (2014), 995-1001. 1

[50] F. Q. Zhang, J. Q. Li, C. W. Zheng, L. Wang, Dynamics of an HBV/HCV infection model with intracellular delay and cell proliferation, Commun. Nonlinear Sci. Numer. Simul., 42 (2017), 464-476. 1

[51] S. Zhang, X. Xu, Dynamic analysis and optimal control for a model of hepatitis $C$ with treatment, Commun. Nonlinear Sci. Numer. Simul., 46 (2017), 14-25. 1 